\title{
Dipole strength distributions from HIGS Experiments
}

\author{
V. Werner ${ }^{1,2, a}$, N. Cooper², P.M. Goddard ${ }^{2,3}$, P. Humby ${ }^{2,3}$, R.S. Ilieva ${ }^{2,3}$, G. Rusev ${ }^{4}$, J. Beller ${ }^{1}$, C. Bernards ${ }^{2}$ \\ B.P. Crider ${ }^{5}$, J. Isaak ${ }^{6,7}$, J.H. Kelley ${ }^{8}$, E. Kwan ${ }^{8,9}$, B. Löher ${ }^{6,7}$, E.E. Peters ${ }^{10}$, N. Pietralla ${ }^{1}$, C. Romig ${ }^{1}$, \\ D. Savran ${ }^{6,7}$, M. Scheck ${ }^{11,12}$, A.P. Tonchev ${ }^{9}$, W. Tornow ${ }^{8}$, S.W. Yates ${ }^{5,10}$, and M. Zweidinger ${ }^{1}$ \\ ${ }^{1}$ Institut für Kernphysik, TU Darmstadt, Schlossgartenstr. 9, 64289 Darmstadt, Germany \\ ${ }^{2}$ WNSL, Yale University, P.O. Box 208120, New Haven, CT 06520, USA \\ ${ }^{3}$ Department of Physics, University of Surrey, Guildford, GU2 7XH, UK \\ ${ }^{4}$ Chemistry Division, Los Alamos National Laboratory, Los Alamos, NM 87545, USA \\ ${ }^{5}$ Department of Physics \& Astronomy, University of Kentucky, Lexington, KY 40506, USA \\ ${ }^{6}$ EMMI and Research Division, GSI, Planckstr. 1, 64291 Darmstadt, Germany \\ ${ }^{7}$ FIAS, Ruth-Moufang-Str. 1, 60438 Frankfurt am Main, Germany \\ ${ }^{8}$ Triangle Universities Nuclear Laboratory, Durham, NC 27708, USA \\ ${ }^{9}$ Physics Division, Lawrence Livermore National Laboratory, Livermore, CA 94550, USA \\ ${ }^{10}$ Department of Chemistry, University of Kentucky, Lexington, KY 40506, USA \\ ${ }^{11}$ School of Engineering, University of the West of Scotland, Paisley, PA1 2BE, UK \\ ${ }^{12}$ SUPA, Scottish Universities Physics Alliance, Glasgow, G12 8QQ, UK
}

\begin{abstract}
A series of photon scattering experiments has been performed on the double-beta decay partners ${ }^{76} \mathrm{Ge}$ and ${ }^{76} \mathrm{Se}$, in order to investigate their dipole response up to the neutron separation threshold. Gamma-ray beams from bremsstrahlung at the S-DALINAC and from Compton-backscattering at HIGS have been used to measure absolute cross sections and parities of dipole excited states, respectively. The HIGS data allows for indirect measurement of averaged branching ratios, which leads to significant corrections in the observed excitation cross sections. Results are compared to statistical calculations, to test photon strength functions and the Axel-Brink hypothesis.
\end{abstract}

\section{Introduction}

The nuclear dipole response, dominated by the giant dipole resonance (GDR), has raised enhanced interest in recent years, mainly due to dipole strength on the lowenergy tail of the GDR. Besides collective excitations in the valence space, such as the magnetic nuclear scissors mode [1-4] or the $1^{-}$member of the quadrupole-octupole coupled multiplet (see, e.g., Ref. [5]), there has been a focus on the so-called pygmy dipole resonance (PDR), which is typically located around 5 to $8 \mathrm{MeV}$ excitation energy (for a recent review on the PDR, see Ref. [6]). A common interpretation of the PDR in nuclei with excess neutrons is the vibration of a proton-neutron core against a neutron skin [7-10]. The amount of the energy-weighted sum rule of the E1 excitation strength which is absorbed in the pygmy resonance is of high interest. To obtain this amount experimentally is difficult. Firstly, the PDR is located on the tail of the GDR, the parametrization of which is not clear at low energies. Therefore, the derivation of proper photon strength functions (PSFs) for the E1 response is highly desirable. Secondly, due to typically high level densities, hence, a high degree of fragmentation of E1 strength in the energy region of interest, a com-

\footnotetext{
a e-mail: vw@ikp.tu-darmstadt.de
}

plete measurement of all E1 excited states is not possible. Thirdly, the often not (directly) observed decay behavior of dipole excited states in the region poses a problem to a proper derivation of their excitation strengths.

In this paper, the focus will be on the present techniques to derive E1 strength distributions from a combination of nuclear resonance fluorescence (NRF) experiments using continuous bremsstrahlung beams in combination with nearly monoenergetic, fully polarized photon beams as available at the High Intensity Gamma-ray Source (HIGS) facility at TUNL, on the campus of Duke University. Results from a series of experiments on the nuclei ${ }^{76} \mathrm{Se}$ and ${ }^{76} \mathrm{Ge}$, performed at the S-DALINAC facility at TU Darmstadt and at HIGS will be discussed. Data have been obtained for low-lying dipole excited states in this candidate pair for neutrino less double-beta $(0 v 2 \beta)$ decay, which is potentially important in the discussion of $\gamma$-ray background in large-scale experiments searching for this rare (if existing) decay mode. In this work, we will focus on the PDR region, and compare the new data to calculations within a statistical approach. 


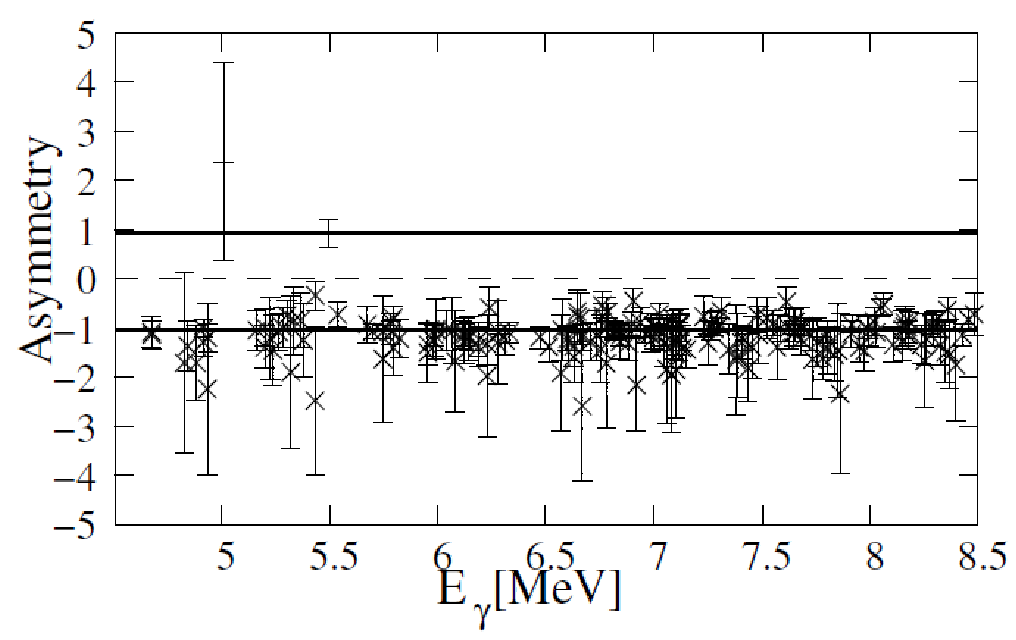

Figure 1. Asymmetries $(A)$ of ground-state transitions from dipole excited states in ${ }^{76} \mathrm{Ge}$. Bars mark the expected limits for positive and negative parity states, the dashed line marks isotropy. E1 (M1) excited negative (positive) parity states have an asymmetry of -1 $(+1)$.

\section{Experiments}

In the following, a brief overview of the performed experiments is given. A more extensive review of the experiments, for the case of ${ }^{76} \mathrm{Se}$, is given in Refs. [11, 12], and a publication for analogous work on ${ }^{76} \mathrm{Ge}$ is forthcoming.

\subsection{Bremsstrahlung}

Experiments using photon beams from bremsstrahlung were conducted at the Darmstadt High Intensity Photon Setup (DHIPS) [13] at the S-DALINAC facility at TU Darmstadt. Electron beams of 5, 7, and $9 \mathrm{MeV}$ in energy for ${ }^{76} \mathrm{Se}$, and $9 \mathrm{MeV}$ only for ${ }^{76} \mathrm{Ge}$ were provided by the injector of the S-DALINAC. The electron beams were converted into continuous-energy photon beams on a thick copper radiator plate, with maximum energies corresponding to the initial electron energies. The photon beams subsequently passed an approximately $1-\mathrm{m}$ long copper collimator before impinging on the respective targets.

Target nuclei were photo-excited with integrated cross sections of

$$
I_{i}^{S}=\left(\pi \frac{\hbar c}{E_{x}}\right)^{2} \frac{2 J_{x}+1}{2 J_{0}+1} \frac{\Gamma_{0} \Gamma_{i}}{\Gamma},
$$

where $E_{x}$ is the excitation energy, $J_{x, 0}$ are the excited-state and ground-state spins, respectively (i.e., $J_{x}=1, J_{0}=0$ here), $\Gamma_{i}$ are partial decay widths from the state $J_{x}$ to a lower-lying state $J_{i}(i=0$ for the ground state) and $\Gamma$ is the total decay width

$$
\Gamma=\sum_{i} \Gamma_{i}=\frac{\hbar}{\tau} .
$$

Absolute integrated cross sections have been measured relative to calibration standards $\left({ }^{27} \mathrm{Al}\right.$ and $\left.{ }^{11} \mathrm{~B}\right)$, which were added to the targets. Details of the NRF technique can be found in Ref. [14]. For most observed states, only the ground-state decay, hence, the corresponding integrated cross section $I_{0}^{S}$, could be measured, since other transitions to lower-lying excited states were masked by the exponentially increasing background toward lower energies. Due to the diminishing photon flux, sensitivity at the highest energies was limited. In addition, no parities were measured in the bremsstrahlung experiment, since the beam was not polarized and Compton polarimeters are typically not sensitive at high $\gamma$-ray energies. These problems have been overcome in the HIGS experiments.

\subsection{Near monoenergetic, polarized beams}

Complementary experiments were performed at the HIGS facility at TUNL. Here, near-monoenergetic photon beams with a beam energy spread of approximately 3\% were produced in a free-electron laser through Comptonbackscattering [15]. The main advantages are the narrow bandwidth of the photon beams and the near $100 \%$ polarization achieved at HIGS. This allows excitations of specific states, or small regions of states, and determination of their parities through simple polarimetry [16]. Background toward low energies still exists, but is largely suppressed as compared to bremsstrahlung experiments. With detectors at $90^{\circ}$ with respect to the beam axis, parallel, i.e., horizontal at HIGS $(h)$, and vertical $(v)$ to the polarization vector of the incoming photons, one obtains an experimental asymmetry in the count rates,

$$
A=\frac{N_{h}-N_{v}}{N_{h}+N_{v}},
$$

where $N_{h, v}$ are the respective observed intensities, $Q$ is an attenuation factor due to finite detector size. Hence, transitions to the ground state from M1 excited states have ideally $A=1$, and from E1 excited states $A=-1$. The factor $Q$ causes an attenuation of those values due to the finite detector solid angles. Figure 1 shows the first results for asymmetries of ground-state decays from dipole excited states in ${ }^{76} \mathrm{Ge}$. Most observed states have negative parity. 


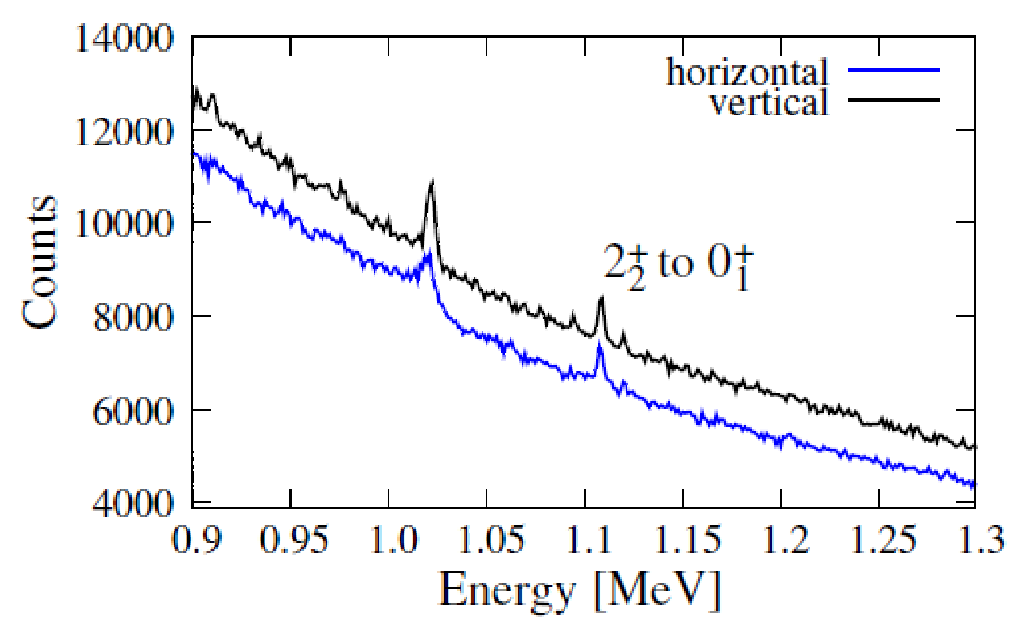

Figure 2. A peak from the $2_{2}^{+} \rightarrow 0_{1}^{+}$transition observed in the horizontal (blue, bottom) and vertical (black, top) detectors, on top of the non-resonant background, far below the beam energy. The peak at $1022 \mathrm{keV}$ stems from $e^{+} e^{-}$annihilation.

In addition to parities, cross sections of newly observed states can be deduced relative to those of neighboring states with already known cross sections (e.g., from the bremsstrahlung experiment), which are covered in the same beam setting. The shape of the photon flux at the target position is simulated using GEANT4, and compared to a spectrum taken with an additional detector at zero degrees, which has been placed into an attenuated beam for each setting.

Not all transitions to lower-lying states were observed in the experiments. However, following Ref. [17] one can assume that most decays pass through the first few excited $2^{+}$states. The observed decays (see Fig. 2 for an example) from those states can then be used to obtain an average branching ratio for decays to excited states over decays to the ground state for each beam setting. This information can then be used to correct the observed cross sections from ground-state decays by the indirectly observed branching via

$$
I_{t o t}^{S}=I_{\Sigma, 0}^{S}\left(1+\sum_{i} \frac{N_{i} W_{0}(\theta)}{N_{\Sigma, 0} W_{i}(\theta)}\right)
$$

where $I_{\Sigma, 0}^{S}$ is the sum of individual integrated groundstate decay cross sections of all states observed within the beam energy window, and $N_{\Sigma, 0}$ are the respective intensities of these transitions with known angular distributions $W_{0} . N_{i}$ are the intensities of the observed $2^{+}$decays to the ground state, with angular distributions $W_{i}$, which are nearly isotropic. Corrections to efficiency calibrations due to non-resonant absorption in the extended target are absorbed in the intensities $N_{i}$. A more detailed description of the procedure can be found in Ref. [12].

\section{Results and discussion}

\subsection{Obtained cross sections}

For ${ }^{76} \mathrm{Se}$, as seen in Fig. 10 of Ref. [12], the low-energy E1 cross sections from the experiments discussed in this paper connect quite smoothly to $(\gamma, n)$ cross section data from Carlos et al. [18]. Some enhancement may exist around $7 \mathrm{MeV}$ in excitation energy, but a conclusion on enhanced E1 strength at low energies depends strongly on the fit function and extrapolation used for the GDR. Two Lorentzians (standard [SLO] and generalized [GLO]) had been fitted to the GDR, giving contradictory conclusions on the presence of enhanced strength [12]. The preliminary results for ${ }^{76} \mathrm{Ge}$ show a very similar behavior, except that no structure appears around $7 \mathrm{MeV}$. In both cases the obtained cross sections follow Lorentzian parametrizations fit to the GDR and the new low-energy data. Only at energies below $6 \mathrm{MeV}$ do the data drop significantly below the Lorentzian parametrizations, as is typically found in a wide range of nuclei.

The lack of any enhancement in the expected PDR region around $7 \mathrm{MeV}$ is surprising. Many isotopes, albeit in different regions, have been investigated to date (see the review in Ref. [6]). Complementary $\alpha$-scattering experiments like, e.g., in Ref. [19], clearly show that the dipole response changes structure from purely isovector at high energies to possessing significant isoscalar components at low, PDR energies. Hence, the lack of an enhancement in the case of the Ge and Se isotopes investigated in this work may point in a new set of directions. One is the potential influence of deformation toward a broadening of the resonance. Another may be a relatively small E1 strength carried by the PDR, so that we do not observe significant (in view of experimental errors) enhancement of E1 strength. A third possibility is that the simple choice of the Lorentzian parametrization masks potential PDR strength in the region of interest. Different Lorentzian parametrizations have been suggested, e.g., in Ref. [20].

Since the precision of data and analysis methods have recently improved vastly, especially through the use of the HIGS facility, we are approaching the situation where we can probe PSFs by comparison of data to statistical calculations. Methods in data analysis and input PSFs vary in recent works (see, e.g., Refs. [21-23]). In the follow- 
ing section we will confront our results with a statistical approach.

\subsection{Statistical calculations}

A new code has been developed within this work to obtain a prediction of photo-cross sections, based on the known level scheme at low energies, on a simulated level scheme above a certain energy threshold involving level densities and a Wigner distribution for level spacings, and on a trial PSF. With this input, average decay widths of dipole excited states to lower-lying states are computed and then varied by Porter-Thomas fluctuations. The Axel-Brink hypothesis $[24,25]$ is assumed to be valid. In order to compare to experiment, a detection limit corresponding to the experimental sensitivity has been imposed on the calculated data. In the following paragraphs, we focus on partial cross sections for ground-state decays only.

Simple assumptions for PSFs have been used to start, namely, the PSF suggested by Kadmenskii, Markushev, and Furman (KMF), which was derived in the low-energy limit, and a double-Lorentzian (SLO) function fitted to data. While the KMF underpredicts data at higher energies, the SLO yields an overprediction at low energies. As a compromise, following the $\mathrm{PhD}$ work of $\mathrm{M}$. Krtička, we choose a PSF from a combination of the KMF and SLO PSFs, using the KMF at low energy, the SLO at high energy, and a linear cross-over from KMF to SLO around the energy where the steep rise in observed cross sections occurs. Figure 3 shows the present status of the statistical calculations in comparison to data from the abovementioned experiments for ${ }^{76} \mathrm{Se}$. The data are integrated over a running bin of $250-\mathrm{keV}$ width.

It is evident from Fig. 3 that the combined KMF/SLO E1 PSF overpredicts data at high energies, unless the experimental detection limit is taken into account (dashed lines). Furthermore, the agreement with data is enhanced if also M1 strength is considered. Since data on M1 excitations in the region of interest is in general sparse, the combined M1 strength observed in the present experiments has been used to derive an M1 strength function (fit to a singleparticle strength function, i.e., a constant value) as input to the calculations. This lowers the calculated values for E1 cross sections at higher energies, and gives good agreement with data.

\section{Conclusions}

A series of NRF experiments, using continuous unpolarized bremsstrahlung beams, as well as near-monoenergetic fully polarized beams from Compton-backscattering, were performed on ${ }^{76} \mathrm{Ge}$ and ${ }^{76} \mathrm{Se}$. The near-final status of the data analysis does not show an enhancement of E1 strength above fitted SLO functions fit to all available E1 response data. Statistical calculations have been performed and show good agreement with data when assuming a hybrid of KMF and SLO E1 PSFs. Note that the inclusion of M1 strength in these calculations significantly enhances

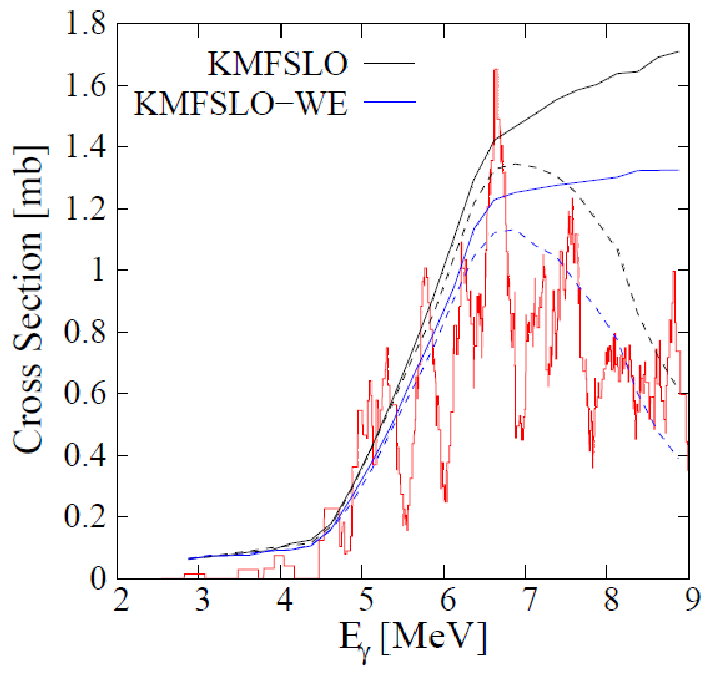

Figure 3. Cross sections, integrated over a running window of $250-\mathrm{keV}$ width, for photo-excitation of $1^{-}$states in ${ }^{76} \mathrm{Se}$ and decay to the ground state from data (red line). The (upper) solid black line shows the result of the statistical calculation considering only the combined KMF/SLO PSF for E1 strength. The (lower) solid blue line includes a Weisskopf estimate for M1 strengths. The respective dashed lines impose the experimental detection limit on the calculations.

agreement with data, and points to the importance of further measurements of M1 strengths at the relevant energies. We stress that there is experimental evidence for a change in the wave functions of E1 excited states from the GDR region toward low energies, corresponding to a PDR. However, the present results show that it may be difficult to quantify the excess of E1 strength due to a PDR. The extraction of such PDR E1 strength largely depends on the choice of PSFs, which are at present dominated by data on the GDR with given uncertainties from conflicting measurements [26]. Data becoming available today, especially from the HIGS facility, start allowing us to constrain PSFs through the use of statistical calculations.

\section{Outlook}

Additional data for both isotopes have been taken at energies below $4 \mathrm{MeV}$, which will give access to collective excitations such as the $1^{+}$scissors mode and quadrupoleoctupole coupled $1^{-}$states. For ${ }^{76} \mathrm{Ge}$, coincidence data taken with the new $\gamma^{3}$ setup [27] will elucidate the decay behavior of such excitations. We also observed a dipole excited state at $3952 \mathrm{keV}$ in ${ }^{76} \mathrm{Ge}$, which is a potential source of background near the expected signal in $0 v 2 \beta$ experiments at $2040 \mathrm{keV}$.

\section{Acknowledgments}

The authors acknowledge support through U.S. DOE grant Nos. DE-FG02-91ER-40609, DE-FG02-97ER41042, and DE-FG02-97ER41033, the German BMBF grant SFB634, the U.S. National Science Foundation grant No. PHY1305801, the UK STFC grant Nos. ST/J500768/1, 
ST/J00051/1, and ST/I005528/1, and the Helmholtz Alliance Program grant No. HA216/EMMI. We further thank E. Grosse for discussions and are grateful to the staff at the S-DALINAC and HIGS facilities for providing excellent experimental conditions.

\section{References}

[1] N. Lo Iudice and F. Palumbo, Phys. Rev. Lett. 41, 1532 (1978); Nucl. Phys. A326, 193 (1979)

[2] F. Iachello and A. Arima, "The Interacting Boson Model" (Cambridge University Press, Cambridge, 1987)

[3] D. Bohle et al., Phys. Lett. B 195, 326 (1987)

[4] A. Richter, Proc. of the Int. Conf. on Nuclear Physics, Florence 1983, ed. P. Blasi and R. A. Ricci, (Tipografica Compositori Bologna, Vol. 2), p. 189

[5] W. Andrejtscheff et al., Phys. Lett. B 506, 239 (2000)

[6] D. Savran, T. Aumann, and A. Zilges, Prog. Part. Nucl. Phys. 70, 210 (2013)

[7] N. Tsoneva and H. Lenske, Phys. Rev. C 77, 024321 (2008)

[8] R. Mohan, M. Danos, and L.C. Biedenharn, Phys. Rev. C 3, 1740 (1971)

[9] P. Van Isacker, M.A. Nagarajan, and D.D. Warner, Phys. Rev. C 45, 13(R) (1992)
[10] N. Paar, D. Vretenar, and P. Ring, Phys. Rev. Lett. 94, 182501 (2005)

[11] N. Cooper et al., Phys. Rev. C 86, 034313 (2012)

[12] P.M. Goddard et al., Phys. Rev. C 88, 064308 (2013)

[13] K. Sonnabend et al., Nucl. Instrum. Methods A 640 , $6(2011)$

[14] U. Kneissl, H.-H. Pitz, and A. Zilges, Prog. Part. Nucl. Phys. 37, 349 (1996)

[15] H.R. Weller et al., Prog. Part. Nucl. Phys. 62, 257 (2009)

[16] N. Pietralla et al., Phys. Rev. Lett. 88, 012502 (2001)

[17] A.P. Tonchev et al., Phys. Rev. Lett. 104, 072501 (2010)

[18] P. Carlos et al., Nucl. Phys. A 258, 365 (1976)

[19] J. Endres et al., Phys. Rev. Lett. 105, 212503 (2010)

[20] A.R. Junghans et al., Phys. Lett. B 670, 200 (2008)

[21] C.T. Angell et al., Phys. Rev. C 86, 051302(R) (2012)

[22] J. Isaak et al., Phys. Lett. B 727, 361 (2013)

[23] G. Schramm et al., Phys. Rev. C 85, 014311 (2012)

[24] D.M. Brink, Ph.D. thesis, Oxford University (1955)

[25] P. Axel, Phys. Rev. 126, 671 (1962)

[26] B.L. Berman et al., Phys. Rev. C 36, 1286 (1987)

[27] B. Löher et al., Nucl. Instrum. Methods A 723, 136 (2013) 
